\title{
Interferência de Trichoderma spp. no crescimento do feijoeiro (Phaseolus vulgaris cv. carioca)
}

\author{
Luciana Zago Ethur \\ Antonio Carlos Ferreira da Silva
}

\section{RESUMO}

O objetivo deste trabalho foi o de avaliar a interferência causada pelo fungo Trichoderma spp. no crescimento do feijoeiro (Phaseolus vulgaris cv. Carioca). Para isso, utilizaram-se três isolados J10, 11 e TSM1 de Trichoderma spp. que foram inoculados em arroz com casca e deixados em câmara climática por quatro dias. Misturaram-se $40 \mathrm{~g}$ desse inóculo em solo autoclavado (vasos com capacidade de $700 \mathrm{~g}$ de solo) antes da semeadura (quatro repetições e quatro plantas por vaso). Quinze dias após a semeadura, mediu-se a altura das plantas, desde o ápice até o colo. Constatou-se diferenças significativas no crescimento das plântulas de feijão. 


\section{SUMMARY}

The objective this work was to evaluate the interference caused by the Trichoderma spp. in the growth of the bean plant (Phaseolus vulgaris cultivar Carioca). Then, it was used three isolates J10, I1 and TSM1 of Trichoderma spp. were inoculated in rice with peel and left in a climatic camera for four days. $40 \mathrm{~g}$ of the inoculum were inoculated in sterili soil (vases with capacity of $700 \mathrm{~g}$ of soil) before after sowing (four repetitions and four plants for vase). After fifteen days of the sowing, the hight the plants, from the apex to the lap. It was verified significant differences in the growth of the bean plants.

\section{INTRODUÇÃO}

Trichoderma spp., que é um fungo natural de solo, tem chamado a atenção dos fitopatologistas devido às suas características de alto nível de competição pelo substrato, capacidade de hiperparasitismo e produção de metabólitos (RAMIREZ et al., 1995), apresentando grande potencial para o controle biológico de fitopatógenos.

A possibilidade de reduzir a dependência de utilização de químicos através de práticas alternativas, como a integração de microrganismos antagônicos e doses reduzidas de fungicidas, tem despertado a atenção de muitos pesquisadores (MELO,1991).

É necessária uma grande gama de experimentação para utilizar-se microrganismos em controle biológico no solo, pois não se tem certeza de que sejam $100 \%$ eficientes e seguros. Há necessidade de estudos pormenorizados no sentido de avaliar os possíveis impactos causados pelo uso dos agentes de controle biológico sobre o ambiente (BETTIOL \& GHINI, 1995).

Trichoderma spp. é um fungo muito estudado e usado em trabalhos de controle biológico e além de todos os mecanismos de um ótimo antagonista tem-se sugerido que pode induzir a um aumento no crescimento de plantas através de um fator que regula o crescimento e a germinação de sementes (MELO, 1991; LUZ, 1991).

Este trabalho teve como objetivo avaliar a interferência causada pelo fungo Trichoderma spp. no crescimento do feijoeiro (Phaseolus vulgaris cv Carioca). 


\section{MATERIAL E MÉTODOS}

Os ensaios experimentais foram desenvolvidos no Laboratório de Microrganismos e Controle Biológico de Fitopatógenos do Departamento de Biologia da Universidade Federal de Santa Maria. Os isolados de Trichoderma spp. foram cedidos pelo mesmo laboratório.

Origem dos isolados de Trichoderma spp.: TSM1 - Plantas de alface em Santa Maria - RS (obtido por SILVA, 1997); I1 - Solo de jardim - Santa Maria-RS (obtido por ROSA \& SILVA, 1998); J10 Solo de estufa/UFSM - Santa Maria-RS (obtido por ROSA \& SILVA, 1998).

Os inóculos dos isolados de Trichoderma spp. foram preparados em arroz com casca, distribuído em três erlenmeyers de $250 \mathrm{~mL}$. Sobre o arroz, foram colocados $10 \mathrm{~mL}$ de água destilada e os erlenmeyers foram deixados em temperatura ambiente por 24 horas para que ocorresse absorção da água pelo arroz. Após, foram levados para esterilização em autoclave, a $120^{\circ} \mathrm{C}(1 \mathrm{~atm})$ por $20 \mathrm{~min}$. A inoculação dos isolados 11, J10 e TSM1 de Trichoderma spp. sobre o substrato (arroz com casca) foi através de discos de BDA de $12 \mathrm{~mm}$ de diâmetro, contendo micélio e conídios. Os erlenmeyers contendo os antagonistas permaneceram em câmara climatizada a $24^{\circ} \mathrm{C}$ por quatro dias, com fotoperíodo de $12 \mathrm{~h}$ claro/12h escuro.

O experimento foi realizado em vasos com capacidade para $700 \mathrm{~g}$ de solo. O solo utilizado foi autoclavado por 2 horas a $120^{\circ} \mathrm{C}(1 \mathrm{~atm})$ para se ter certeza que a interferência no crescimento do feijoeiro ocorreria pela ação dos isolados de Trichoderma spp.

Depois de preenchidos os 16 vasos com o solo, foi feita a inoculação dos 3 isolados de Trichoderma spp. (com 4 repetições). No momento da semeadura (10 sementes por vaso) de feijão misturaram-se $40 \mathrm{~g}$ do inóculo superficialmente ao solo, deixando-se 4 vasos sem o antagonista para controle. Os vasos foram mantidos em temperatura de $21^{\circ} \mathrm{C}$ e fotoperíodo acionado $(12 \mathrm{~h}$ claro/12 h escuro).

Após sete dias da semeadura, fez-se o desbaste deixando-se 4 plantas por vaso, e aos quinze dias, mediu-se a altura (do ápice até o colo) das plantas.

\section{RESULTADOS E DISCUSSÃO}

Os 3 isolados de Trichoderma spp. tiveram comportamento diferenciado, sendo que o TSM1 desenvolveu abundante 
micélio esbranquiçado (sem esporulação aparente) e os isolados I1 e J10 apresentaram menor desenvolvimento micelial (aparente) e elevada esporulação (esverdeada). Segundo pesquisa realizada por SILVA \& MELO (1986) a esporulação dos isolados de Trichoderma pode variar dependendo de fatores como a luminosidade.

TABELA 1 - Avaliação do crescimento das plântulas de feijoeiro em centímetros (do colo até a ponta da haste), 15 dias após a semeadura. Santa Maria - RS, 1999.

\begin{tabular}{cc}
\hline ISOLADOS DE Trichoderma & ALTURA DO FEIJOEIRO $(\mathrm{cm})$ \\
\hline CONTROLE & $28.525002 \mathrm{a}$ \\
TSM1 & $31.299999 \mathrm{a}$ \\
J10 & $15.100000 \mathrm{~b}$ \\
I1 & $17.674999 \mathrm{~b}$ \\
\hline
\end{tabular}

${ }^{*}$ Média de 04 plantas por vaso.

Médias seguidas de mesma letra minúscula não diferem entre si (Teste de Tukey - 5\%).

Ocorreu diferenciação quanto ao crescimento das plântulas de feijoeiro inoculadas com os isolados de Trichoderma spp. A diferença encontrada entre o controle e os vasos com o isolado TSM1 não foi significativa, mas encontrou-se significância quando comparou-se o controle com os isolados J10 e TSM1 de Trichoderma spp. (Tabela 1), demonstrando que Trichoderma spp. é capaz de interferir no crescimento do feijoeiro.

A eficácia de Trichoderma no crescimento de vegetais foi mencionada por Windham et al. (1986) e Martins (1988) apud MELO (1991), onde isolados de Trichoderma spp. induziram o crescimento de plantas e estimularam a germinação de sementes. Da mesma forma, MENEZES (1992) constatou efeito positivo no crescimento do feijoeiro com o uso de Trichoderma, em solo esterilizado e não esterilizado. O mesmo efeito positivo foi declarado por PIMENTEL et al. (1994) em plantas de cacau, e por PORFÍRIO-SILVA e HOMECHIN (1992) em plântulas de tomateiro. 
No entanto, ASSIS (1994) encontrou redução na germinação de sementes de mamona devido ao uso de Trichoderma spp. Essa redução na germinação e no crescimento de certas plantas deve ser levada em consideração, pois a principal forma de utilização de Trichoderma spp. para controle biológico ocorre com inoculação no solo (FERNÁNDEZ-LARREA, 1995).

\section{CONCLUSÕES}

Constatou-se que ocorreu interferência dos isolados de Trichoderma spp. no crescimento do feijoeiro, pois o tratamento com os isolados $\mathrm{J} 10$ e $\mathrm{I} 1$ apresentaram menor altura das plântulas de feijoeiro comparados com os tratamentos controle e do isolado TSM1, os quais não diferiram entre si. 


\section{REFERÊNCIAS BIBLIOGRÁFICAS}

ASSIS, T.C. Controle biológico de microrganismos presentes em sementes de mamona (Ricinus comunis). Fitopatologia Brasileira, v.19, Suplemento, p. 269, agosto de 1994.

BETTIOL, W., GHINI, R. Controle biológico. Cap. 36. In: BERGAMIN FILHO, A., KIMATI, H., AMORIN, L. Manual de fitopatologia. 3. ed. São Paulo: Agronômica Ceres, 1995. 919 p. 2v. v1.

FERNANDEZ-LARREA, O.V. Microorganismos entomopatógenos y antagonistas. Posibilidades de produción. C. de La Habana : INISAV, 1995. 15 p. (Boletim Técnico, 1).

LUZ, W.C. da. Controle biológico das doenças na espermosfera. cap. 3. In: BETTIOL, W. (org.) Controle biológico de doenças de plantas. Jaguariúna : EMBRAPA-CNPDA, 1991.(EMBRAPA-CNPDA. Documentos, 15).

MELO, I.S. de. Potencialidades de utilização de Trichoderma spp. no controle biológico de doenças de plantas. cap. 9. In: BETTIOL, W. (org.) Controle biológico de doenças de plantas. Jaguariúna : EMBRAPA-CNPDA, 1991. (EMBRAPA-CNPDA. Documentos, 15).

MENEZES, M. Avaliação de espécies de Trichoderma no tratamento de sementes de feijão e do solo, visando o controle de Macrophomina phaseolina. Fitopatologia Brasileira, v.17, n.2, p. 159, agosto de 1992.

PIMENTEL, C.P.V., CORAL, F.J., LUCON, C.M.M., FARIA, R.G. Efeito de Trichoderma viride sobre Phytophthora spp. Fitopatologia Brasileira, v.19, Suplemento, p. 301, agosto de 1994.

PORFÍRIO-SILVA, Z., HOMECHIN, M. Pseudomonas spp. e Trichoderma spp.: promotores de crescimento de plântulas de tomateiro. Fitopatologia Brasileira, v.17, n.2, p. 217, agosto de 1992.

RAMIREZ, I.S., LÓPEZ, M.O., GARCÍA, D., et al. Trichoderma harzianum (cepa A 34): un biopreparado de amplio espectro para micopatologías del tomate y del pimiento. C. de La Habana : INISAV, 1995. 36 p. (CID-INISAV Boletín Técnico, 3).

ROSA, C.R.E, SILVA, A.C.F. Avaliação de isolados de Trichoderma spp. visando o controle de Sclerotinia sclerotiorum. In: JORNADA ACADÊMICA INTEGRADA, 13. Santa Maria - RS, 1998. Anais... Universidade Federal de Santa Maria, 1998. 
SILVA, A.C.F. da. Uso da radiação gama para obtenção de mutantes de Trichoderma harzianum Rifai e Trichoderma viride Pers.: Fr. com capacidade melhorada no controle ao Sclerotinia sclerotiorum (Lib.) de Bary. Tese de Doutorado. (Centro de Energia Nuclear na Agricultura - USP) Piracicaba : Universidade de São Paulo, 1997. SILVA, A.C.F. da, MELO, I.S. Influência da luz no crescimento micelial e produção de esporos em diferentes linhagens de Trichoderma spp. In: REUNIÃO SOBRE CONTROLE BIOLÓGICO DE DOENÇAS DE PLANTAS, 1. Anais... Piracicaba, Fundação Cargil ,1986.

LUCIANA ZAGO ETHUR

Especialista em Biologia

CCNE/UFSM

ANTONIO CARLOS FERREIRA DA SILVA

Departamento de Biologia

CCNE - UFSM

Santa Maria, RS 
88 Ciência \& Natura, Santa Maria, 23: 81 - 87, 2001. 